\title{
Outcomes of multiple myxoma resections with carney complex
}

\author{
Juan A Siordia, Amanda Garlish \\ Department of Surgery, University of Arizona Medical Center, University of Arizona, Arizona, United States
}

Received: July 17, 2015

DOI: $10.5430 /$ css.v1n1p50
Accepted: August 5, 2015

Online Published: August 17, 2015

\begin{abstract}
Introduction: Carney Complex is a rare multiple endocrine neoplasia with cardiac myxomas being the most common cause of death among affected patients. One must consider how many heart surgeries a patient may acquire before the treatment itself becomes a significant risk factor. The use of an intra-aortic filter during cardiac surgeries is recommended, and heart transplantation treatment for Carney Complex patients is proposed.

Presentation of case: A 24-year-old female presented with lightheadedness and was found to have a cardiac myxoma on the left atrium. The patient underwent myxoma resections on four separate occasions. The patient underwent a fifth cardiac myxoma resection. However, three days post-operatively, the patient experienced right-sided hemiplegia and imaging showed 99\% left carotid artery occlusion. The patient was deemed unqualified for neurosurgical intervention and the patient died 5 days post-operatively.

Discussion: Cardiac surgery can become detrimental with multiple surgical resections. With increased number of cardiac surgeries and increased risk for morbidities postoperatively, Carney Complex patients should have an intra-aortic filter to lower the risk of stroke.

Conclusions: Intra-aortic filter usage is recommended during surgical resection of cardiac tumors to capture emboli before they travel into the systemic circulation. Heart transplant may prove beneficial to Carney complex cases, however neither the optimal number of cardiac operations nor the timing of heart transplantation in the disease are documented in literature, limiting evidence for solid therapeutic guidelines.
\end{abstract}

Key Words: Carney complex, Myxoma, Cardiac surgery, Intra-aortic filter

\section{INTRODUCTION}

Carney Complex is a rare multiple endocrine neoplasia that presents with a variety of affected organ systems. The complex was described in 1985 after Carney et al. discovered the pattern of cardiac myxomas along with skin pigmentation and primary pigmented nodular adrenocortical disease (PPNAD). ${ }^{[1]}$ The disease presents with spotty skin pigmentation (77\%), cardiac myxomas (53\%), skin myxomas (33\%), PPNAD (26\%), and acromegaly (10\%); depending on the gender of the patient, it may also produce large-cell calcifying Sertoli cell tumors (LCCSCT) (33\% of male patients) and breast ductal adenoma (3\% of female patients). ${ }^{[2]}$ The disease presents with myxomatous changes - abnormal proliferation of mucin and collagen - in various organ systems.

Cardiac myxomas are the most common cause of death among patients with Carney complex. This includes heartrelated issues (57\%), cardiac myxoma obstruction (25\%), emboli (12\%), heart surgery complications (10\%), cardiomy-

*Correspondence: Juan A Siordia; Email: jas@email.arizona.edu; Address: 329 N. Norris Ave., Tucson, Arizona, United States. 
opathy $(4 \%)$, or fatal cardiac arrhythmias $(6 \%) .{ }^{[2]}$ Presentation of myxomas on echocardiography demands resection. However, the complex is associated with multiple presentations. One must consider how many heart surgeries a patient may acquire before the treatment itself becomes a significant risk factor.

Perioperative mortality of cardiac myxoma removal is minute, but some complications may still occur postoperatively, including supraventricular arrhythmias, embolic strokes, decreased cardiac output, and myxoma recurrence. ${ }^{[3,4]}$ Embolic events are a known complication from cardiac myxomas. There is a $30 \%$ rate of association of systemic emboli manifestations with left atrial myxomas alone. ${ }^{[5]}$ The Embol-X intraaortic filter (Edwards Lifesciences In., Irvine, California) is an aortic cannulation device that prevents cardiac embolic phenomenon from cardiac surgery. Showing a $74 \%$ reduction in cerebral injury in highrisk patients after cardiac surgery, ${ }^{[6]}$ it may serve effect in the surgeries performed in Carney complex patients.

Recurrence of cardiac myxomas is also marked at $10 \%$ $21 \%,{ }^{[7,8]}$ either from incomplete resection or Carney complex. The treatment for recurrent myxoma is redo-cardiac surgery. However, multiple cardiac surgeries can cause cardiac dysfunction due to repeated periods of ischemia in addition to iatrogenic fibrosis via surgical incisions on the cardiac musculature. Therefore, other therapies may be considered. Cardiac transplantation is rarely performed for benign cardiac neoplasms and has not been documented for the use on Carney complex patients. Studies have not compared the use of transplantation as a substitute to resection therapy after multiple cardiac myxoma resections in Carney complex. ${ }^{[9]}$

\section{Case presentation}

A 24-year-old female presents to the hospital with a chief complaint of lightheadedness on year 2006. Past medical history was significant for hypothyroidism and diabetes. Physical exam was significant for multiple ephelides on the face and a heart murmur. An echocardiogram demonstrated a cardiac myxoma on the left atrium. Resection of the myxoma occurred via a median sternotomy. After initiation of cardiopulmonary bypass, the Embol-x intra-aortic filter was deployed within the aorta to capture embolic and myxoma debris. After aortic cross-clamping, dissection of the heart exposed the myxoma in the left atrium, allowing adequate visualization for ensured complete removal.

The patient returned with multiple presentations of cardiac myxoma in years 2008, 2010, and 2011. Each myxoma was located on a different area: right atrium on 2008 and left ventricle on both 2010 and 2011. Resection procedure for

Published by Sciedu Press each presentation was similar to that described in the first operation; removal of adhesions was also performed with each operation.

Lab values on the third visit showed a significantly increased amount of growth hormone; the presentation was subclinical. Carney Complex was diagnosed on the third visit in 2010. On the third operation, extensive endocardial ablation with cautery was performed on suggestive neoplastic foci after endoscopic visualization of all four heart chambers.

The patient presented with a fifth cardiac myxoma in the left ventricle on 2013. Discussion on treatment led to the suggestion of heart transplantation due to multiple myxoma presentations, the genetic pattern, and multiple redo cardiac operations.

The patient went to another hospital to inquire heart transplantation. Previous operative notes were analyzed and another echocardiogram was performed. Echocardiogram and cardiac MRI showed two large, penduculated myxomas and a thrombus (see Figures 1 and 2). The left ventricular ejection fraction was measured at $41 \%-47 \%$. The diagnosis switched to incomplete cardiac myxoma resection and a 5 th myxoma resection was scheduled instead of heart transplantation.

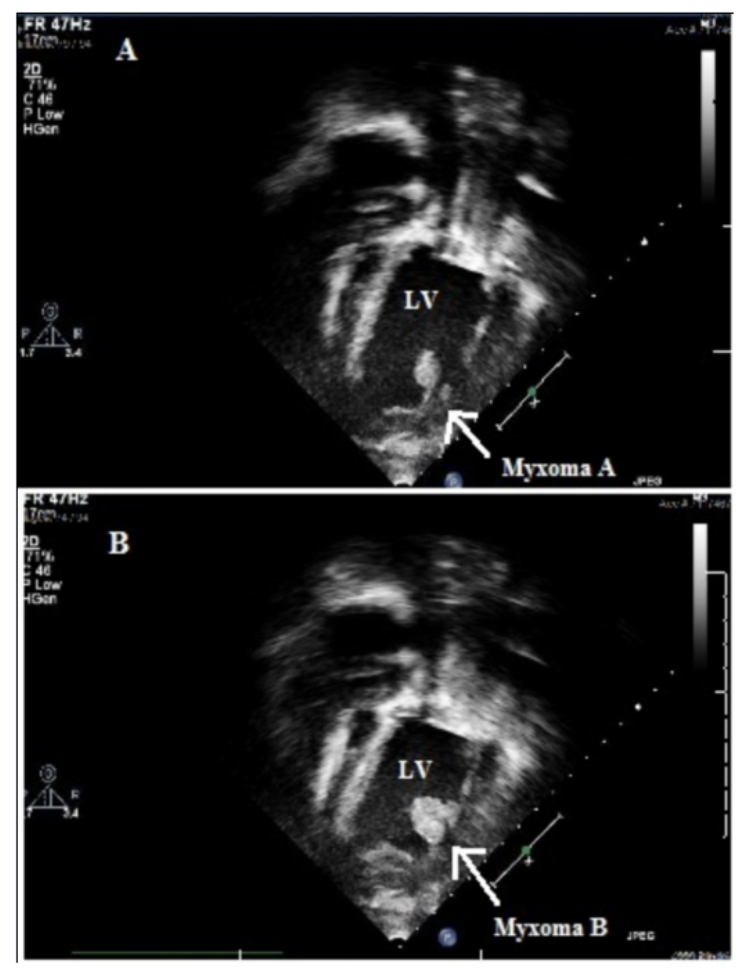

Figure 1. Multiple (3+) large, peduncalated and fimbriated mobile masses were found near the left ventricular apex. The two most prominent measured $1.4 \mathrm{~cm} \times 2.4 \mathrm{~cm}(\mathrm{~A})$ and $1.4 \mathrm{~cm} \times 2.6 \mathrm{~cm}(\mathrm{~B})$. Both appeared affixed to apical lateral $\mathrm{LV}$ wall via thin mobile stalks. 


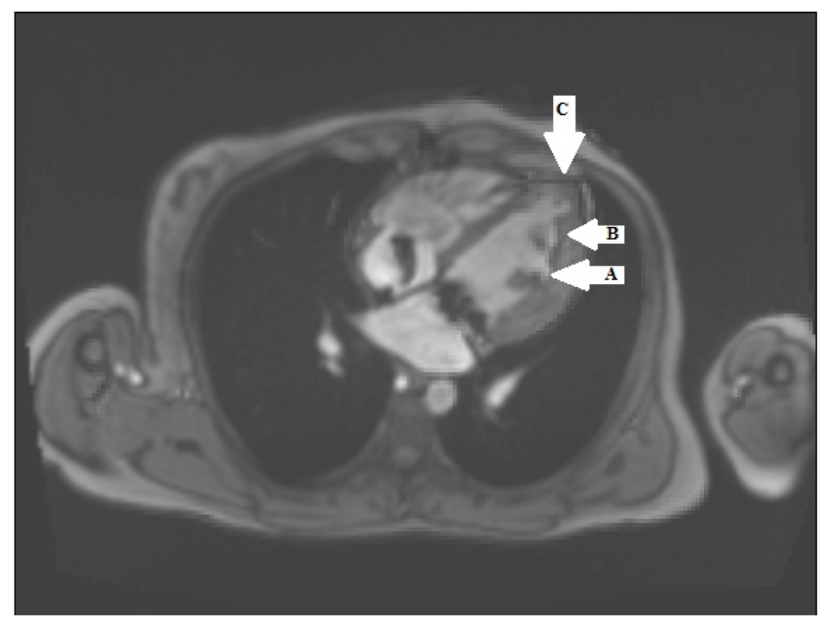

Figure 2. Cardiac MRI on 5th presentation reveals two pedunculated cardiac myxomas (A, B) found within the left ventricle. A left ventricular aneurysm with small thrombus (C) is also visualized at the apex of the chamber.

Pre-operatively, the patient was anticoagulated with heparin due to the evidence of thrombi. After imaging suggested the disappearance of clots, the operation commenced. After going on the heart-lung machine, adhesions were removed around the left ventricle. Multiple neoplastic foci and a thrombus were discovered upon entry. After removal of the thrombus and tumors, the ventriculotomy was closed and the aortic cross-clamp and cardiopulmonary bypass were removed appropriately. Of note, the Embol-x intra-aortic filter was not used during the procedure.

Three days post-operatively, the patient awoke with rightsided hemiplegia. CT scan of the head and neck revealed a 99\% occlusion of the left carotid artery suggestive of embolus from the cardiac surgery case. Neurosurgery deemed the patient unqualified for intervention due to massive prolonged ischemia of the cerebrum. After discussion with the family, the ventilator was removed and the patient died five days postoperatively at the age of 31 years.

\section{Discussion}

Carney Complex (CNC) is an autosomal dominant genetic disease that presents early in life. The genetic defect is found in chromosome 17q2 (CNC1 gene), also known as the PRKAR1A gene. The mutation causes an abnormality in the 1-alpha subunit of protein kinase A, which is responsible for multiple hormonal and intracellular signaling pathways. ${ }^{[9]}$ Diagnosis is achieved with the presentation of 2 major criteria (including skin pigmentation, cardiac myxoma, skin myxoma, and PPNAD); or one major criterion plus evidence of the genetic mutation. ${ }^{[2]}$ Evidenced by the case, cardiac myxomas present at a mean age of 24 years; this is unlike sporadic myxomas that present at a mean age of 51 years. $^{[1]}$
Cardiac myxomas are known to cause abnormalities including valvular pathology, pulmonary congestion, and embolic formation. Congestive heart failure $(68 \%)$ and cerebral strokes $(27 \%)$ are commonly associated with presenting symptoms. ${ }^{[10,11]}$ The mass effect of the myxoma causes obstruction through the heart valve in addition to turbulent blood flow. While the obstructive capabilities are commonly presented as syncope, the turbulence in blood flow contributes to the formation of thromboembolic formation via the principles of Virchow's triad. Due to these possible complications, in addition to the recurrence of myxomas found in Carney complex patients, it is suggested to perform annual echocardiograms in afflicted patients. ${ }^{[9]}$ Detection of a cardiac myxoma prompts surgical excision to avoid the high risk of embolization and stroke. ${ }^{[12]}$

Supported by this case, intra-aortic filter usage is recommended during the operation to capture emboli before they travel into the systemic circulation. Usage of the Embol$\mathrm{X}$ intraaortic filter during cardiac surgery diminished postoperative stroke and embolic events significantly. ${ }^{[13,14]}$ Sobieski et al. demonstrated a $94.5 \%$ rate of embolic matter capture with the filter $(\mathrm{N}=146 ; 74$ patients received the filter and 72 captured emboli matter). ${ }^{[14]}$ It is also marked with a $74 \%$ reduction of cerebral vascular infarcts within high-risk patients. A decrease in renal complications from $24 \%$ to $14 \%$ has also been reported with the use of the filter. ${ }^{[6]}$

Although resection is the current treatment for cardiac myxoma, Carney complex is complicated with multiple cardiac myxoma recurrences. Recurrence of cardiac mxyomas is ranged between $10 \%$ and $21 \% .{ }^{[7,8]}$ The present case involved the evolution of mxyomas in three different chambers. Resection of a cardiac myxoma does present with possible post-opeartive complications, including supraventricular arrhythmias such as atrial fibrillation, embolic strokes, and decreased cardiac output. ${ }^{[3,14]}$ Long-term post-operative complications include those from congestive heart failure or myocardial infarction. ${ }^{[4]}$ Additional cardiac myxoma resections may exacerbate these post-operative complications. A heart is believed to only sustain a limited amount of operations before iatrogenic myocardial fibrosis begins to significantly affect the ejection fraction and cardiac index. Heart transplantation may prove beneficial after a certain amount of resections. ${ }^{[15,16]}$ A heart transplant patient has a mean survival of 10 years; $27 \%$ of patients also live 20 years posttransplantation. ${ }^{[17]}$ The use of cardiac transplantation for any primary cardiac tumor is not recommended since there is a higher 10-year mortality rate with transplantation (25\%) than resections $(2.75 \%) .{ }^{[18]}$ However, there are no studies comparing the effects of transplantation versus resection after multiple cardiac surgeries. 


\section{REFERENCES}

[1] Carney JA, Gordon H, Carpenter PC, et al. The complex of myxomas, spotty pigmentation, and endocrine overactivity. Medicine (Baltimore). 1985; 64: 270-83. http://dx.doi.org/10.1097/0 0005792-198507000-00007

[2] Stratakis CA, Kirschner LS, Carney JA. Clinical and molecular features of the carney complex: diagnostic criteria and recommendations for patient evaluation. J Clin Endocrinol Metab. 2001; 86: 4041-6. PMid: 11549623. http://dx.doi.org/10.1210/jcem.86.9.7 903

[3] Sellke FW, Lemmer JH, Vandenberg BF, et al. Surgical treatment of cardiac myxomas: long-term results. Ann Thorac Surg. 1990; 50: 557-61. http://dx.doi .org/10.1016/0003-4975(90) 90189 $-\mathrm{D}$

[4] Garatti A, Nano G, Canziani A, et al. Surgical excision of cardiac myxomas: twenty years experience at a single institution. Ann Thorac Surg. 2012; 93: 825-31. PMid: 22296980. http: //dx.doi.org/10.1016/j.athoracsur.2011.11.009

[5] Pinede L, Duhaut P, Loire R. Clinical presentation of left atrial cardiac myxoma. A series of 112 consecutive cases. Medicine (Baltimore). 2001; 80: 159-72. http://dx.doi.org/10.1097/00005792-2 00105000-00002

[6] Van Mieghem NM, Schipper ME, Ladich E, et al. Histopathology of embolic debris captured during transcatheter aortic valve replacement. Circulation. 2013; 127: 2194-201. PMid: 23652860. http: //dx.doi.org/10.1161/CIRCULATIONAHA.112.001091

[7] Turhan S, Tulunay C, Altin T, et al. Second recurrence of familial cardiac myxomas in atypical locations. Can J Cardiol. 2008; 24: 715-6. http://dx.doi.org/10.1016/S0828-282X (08)70671-8

[8] Okamoto T, Fujisaki H, Horikoshi Y, et al. A familial case of multiple recurrent cardiac myxomas. Jour Cardiol Cases. 2013; 8: 142-4. http://dx.doi.org/10.1016/j.jccase.2013.07.001
[9] Siordia JA. Medical and surgical management of Carney complex. J Card Surg. 2015; 30: 560-7. PMid: 25996461. http://dx.doi.o rg/10.1111/jocs. 12575

[10] Chen M, Wang JH, Chao SF, et al. Cardiac myxoma originating from the anterior mitral leaflet. Jpn Heart J. 2003; 44: 429-34. PMid: 12825810. http://dx.doi.org/10.1536/jhj. 44.429

[11] Butany J, Nair V, Naseemuddin A, et al. Cardiac tumours: diagnosis and management. Lancet Oncol. 2005; 6: 219-28. http: //dx.doi.org/10.1016/S1470-2045(05)70093-0

[12] Silverman NA. Primary cardiac tumors. Ann Surg. 1980; 191: 127 PMid: 7362282. http://dx.doi.org/10.1097/00000658-198 002000-00001

[13] Christenson JT, Vala DL, Licker M, et al. Intra-aortic filtration: campturing particulate emboli during aortic cross-clamping. Tex Heart Inst J. 2005; 32: 515-21. PMid: 16429895.

[14] Sobieski MA, Pappas PS, Tatooles AJ, et al. Embol-x intra-aortic filtration system: capturing particulate amboli in the cardiac surgery patient. J Extra Corpor Technol. 2005; 37: 222-6. PMid: 16117464.

[15] DiBernardo LR, Kirshbom PM, Skaryak LA, et al. Acute functional consequences of left ventriculotomy. Ann Thorac Surg. 1998; 66: 159-65. http://dx.doi.org/10.1016/S0003-4975(98)0 0376-2

[16] Machiraju VR. Redo cardiac surgery. J Card Surg. 2004; 19: 283. ht tp://dx.doi.org/10.1111/j.0886-0440.2004.4050_11.x

[17] Stehlik J, Edwards LB, Kucheryavaya AY, et al. The registry of the international society for heart and lung transplantation: 29th official adult heart transplant report-2012. J Heart Lung Transplant. 2012; 31: 1052-64. PMid: 22975095. http://dx.doi.org/10.1016/j .healun.2012.08.002

[18] Padalino M, Vida VL, Tonello M, et al. Surgery for primary cardiac tumors in children: early and late results in a multi-center European congenital heart surgeons association study. Circ. 2010; 122: 22-30. 